\title{
Explanation in Biology: Reduction, Pluralism, and Explanatory Aims
}

\author{
Ingo Brigandt
}

Department of Philosophy

University of Alberta

2-40 Assiniboia Hall

Edmonton, AB T6G 2E7

Canada

Email: brigandt@ualberta.ca

Tel: +1 780-492-3307 ext. 12

Fax: +1 780-492-9160

\begin{abstract}
This essay analyzes and develops recent views about explanation in biology. Philosophers of biology have parted with the received deductive-nomological model of scientific explanation primarily by attempting to capture actual biological theorizing and practice. This includes an endorsement of different kinds of explanation (e.g., mathematical and causal-mechanistic), a joint study of discovery and explanation, and an abandonment of models of theory reduction in favor of accounts of explanatory reduction. Of particular current interest are philosophical accounts of complex explanations that appeal to different levels of organismal organization and use contributions from different biological disciplines. The essay lays out one model that views explanatory integration across different disciplines as being structured by scientific problems. I emphasize the philosophical need to take the explanatory aims pursued by different groups of scientists into account, as explanatory aims determine whether different explanations are competing or complementary and govern the dynamics of scientific practice, including interdisciplinary research. I distinguish different kinds of pluralism that philosophers have endorsed in the context of explanation in biology, and draw several implications for science education, especially the need to teach science as an interdisciplinary and dynamic practice guided by scientific problems and explanatory aims.
\end{abstract}

Keywords: biology, explanation, integration, mechanisms, pluralism, reduction, scientific aims, scientific practice, scientific problems 


\section{Introduction}

While scientific explanation is one of the core issues in contemporary philosophy of science, in the first half of the $20^{\text {th }}$ century explanation was not universally seen to be of philosophical concern. Surprising to us, back then it was not always taken for granted that science puts forward explanations. It was uncontroversial that science uncovers laws, confirms hypotheses, and makes predictions. It can be scientifically established that the laws of physics hold, but what about an alleged explanation of why the laws of physics hold? Rather than accepting that the basic principles of physics just obtain and cannot be explained by any more fundamental laws, such a why-question appears to ask for some ulterior rationale for a cosmological order. An explanation would have to appeal to some metaphysical or theological reasons (e.g., a divine being having set up the laws and constants of physics such that intelligent life can evolve), which appear to be illicit, or at any rate not within the scope of science. According to this past view, science predicts events, but it does not endeavor to explain phenomena or answer why-questions.

The seminal account of scientific explanation put forward by Carl Hempel and Paul Oppenheim made explanation an issue for philosophy of science by showing how some explanations can very well be scientific (Hempel and Oppenheim 1948). On their account, any scientific explanation is a logical argument, where the explanandum (a statement expressing the fact to be explained) logically follows from the explanans (a set of statements expressing the facts that provide the explanation). The main condition of the Hempel-Oppenheim account is that the explanans contains at least one law-like statement (apart from other statements such as initial and boundary conditions that ensure that the specific explanandum follows). Such a logical argument is a scientific explanation as the phenomenon to be explained is shown to result from the laws of nature, which is why this account of explanation is often called the covering law 
model of explanation (phenomena fall under laws). Explanations as logical arguments are analogous to scientific predictions, demystifying explanation and showing it to be scientific.

The Hempel-Oppenheim account of explanation was developed within the philosophical tradition of logical positivism. Many views in the philosophy of science, which as a discipline grew to a large extent out of logical positivism during the 1950s and 60s, originated by modification and even rejection of earlier logical positivist doctrines. Philosophical accounts of explanation are no exception. The Hempel-Oppenheim account of explanation was criticized and largely abandoned. What is of particular interest is that it was not replaced by a unique account of explanation; rather, different models of explanation have been proposed. As discussed in the next section, this is particularly true in the philosophy of biology, where it is generally acknowledged that different types of explanation (e.g., statistical explanations and causalmechanistic explanations) exist across biology. As a result, philosophers of biology usually endorse some form of pluralism about the character of scientific explanation.

A second departure from the framework of logical positivism germane to the issue of explanation is the philosophical relevance of scientific practice and discovery. Some of logical positivism (and the early philosophy of science growing out of it) endorsed a strict separation between the context of discovery and the context of justification. Justification (confirmation) was seen as a rational process because once a hypothesis is formulated there are objective ways of determining the degree to which it is supported by available evidence. Discovery - the way in which a scientist invents a hypothesis — in contrast, was seen as a psychological process which need not be rational. For instance, August Kekulé claimed to have come up with the chemical structure of benzene upon having a day-dream of a snake seizing its own tail. Despite this nonrational mode of discovery, the hypothesis could be subsequently confirmed by objective means. 
This traditional view that confirmation but not discovery is of concern to philosophy (the latter being an issue for psychology and sociology) is rejected by most contemporary philosophers of biology. Instead, experimental discovery and the generation of ideas are seen as an integral part of science; biological theorizing is closely tied to biological practice. This is also relevant to explanation in biology, as debates on reductive explanation illustrate (Section 3). Philosophical accounts of reduction of the 1960s focused on the knowledge of one scientific field being in principle reducible to a more fundamental theory. However, critics wondered why this would be relevant to philosophically understanding biology in practice. This attention to actual biological practice (including discovery) led many philosophers of biology to abandon theory reduction and rather endorse pluralism - a pluralism about the structure and overall character of biology. One fruitful way to go beyond reduction is to philosophically study integrative explanations and interdisciplinary research across different biological disciplines. Teaching how interdisciplinarity works is also relevant for science education, so that in Section 4 I lay out (among other things) an epistemology of integration that views interdisciplinary explanation as coordinated by scientific problem agendas.

Section 5 turns to the philosophical relevance of explanatory aims. Many scientific models make idealizations, abstracting away from many facts. While this may limit the applicability of the model, it can be very advantageous given that any model is used for a specific explanatory aim. I discuss differing visions about the centrality of genes in explanations of evolution and development. These differing visions turn out to be compatible once it is clarified that they actually concern different explanatory questions, yet conflict results if scientists fail to realize that different approaches pursue different explanatory aims. Idealizations made for certain explanatory purposes can yield a form of pluralism that is much stronger than the varieties mentioned so far, as it maintains that some different explanatory models pertaining to a 
phenomenon cannot be integrated. Finally, explanatory aims and values are of philosophical relevance because they account for the dynamics of science-in line with a philosophy of biology that is concerned with understanding biological practice.

Throughout the essay I point out what implications these recent philosophical considerations about explanation have for the teaching of biology. Science education primarily consists in presenting the content of scientific theories, but in line with the recent philosophical emphasis on practice it is equally important to convey to students that science is an inquiry-based process, and how this dynamic inquiry is guided by scientific questions and explanatory aims. Teaching the practice of science is a very challenging task for science education, which is augmented by the complexity of contemporary biology. But given that the nature of science is to constantly revise knowledge, teaching how science as an investigative practice operates is essential for making students understand how scientific knowledge is modified and why and how new explanations are developed. Section 6 summarizes my overall philosophical discussion and the lessons for biology education.

\section{Pluralism about the Character of Scientific Explanation}

According to Hempel’s logical positivist account, an explanation is a special type of logical argument, where the premises (the explanans) contain a statement representing a law, or at least a law-like generalization. The original deductive-nomological model (DN model) assumed that such a logical argument was a valid deductive argument (Hempel and Oppenheim 1948). Thus, the explanans deductively entails the explanandum, i.e., the situation to be explained, which could be a particular fact or a generality (in the latter case a generality is explained by a more general law). While this account of explanation was initially widely accepted, counterexamples 
to it were developed by several philosophers. Take for instance the 'John and the birth control pills’ example. A man called John has regularly taken birth control pills. A putative explanation of why John failed to get pregnant asserts that (i) John has taken birth control pills and that (ii) it is a law-like regularity that all men who take birth control pills do not get pregnant. These two premises are true, and from them it deductively follows that John is not pregnant, so this has to be counted as an explanation by the DN model. Yet it is clear that this is not an explanation, as John did not fail to get pregnant because he took birth control pills.

What is wrong with the logical positivist construal of explanation could be more clearly articulated in response to Hempel's later account of inductive-statistical explanations, which added IS explanations as a second type to DN explanations. An IS explanation has the same characteristics as a DN explanation, except that it is an inductive argument, so that the explanans does not guarantee the truth of the explanandum, but makes the explanandum very likely (Hempel 1965). Wesley Salmon (1971) objected that this misses the nature of statistical explanation. The considerations adduced in the explanans need not make the explanandum likely, rather they have to make the explanandum more likely than alternative circumstances would make. A medical drug may not guarantee the recovery of a patient, but it can still be efficacious if it results in a higher recovery rate than in untreated patients. One can explain why a person got disease symptoms by pointing out that she has a certain genetic variant, even though only $8 \%$ of persons with the variant will actually get the genetic disease-for having this genetic variant raises the probability of getting the disease by a fourfold from $2 \%$ to $8 \%$. This shows why even the DN model is faulty. In the 'John and the birth control pills' example, we have a deductively valid inference from laws, yet it is not an explanation as John taking birth control pills does not make him failing to become pregnant more likely than him not having taken any birth control. Rather than making the truth of the explanandum probable, in a scientific 
explanation the explanans has to adduce factors that are explanatorily relevant. Salmon made this point by claiming that unlike predictions (which show that a certain event must happen or is likely to happen), explanations are not arguments, neither deductive nor inductive arguments. ${ }^{1}$ As a replacement to Hempel’s framework, he put forward his detailed model of statistical explanation, according to which explanations invoke factors that are statistically relevant, i.e., they make the explanandum more likely than alternative conditions would make (Salmon 1971).

Later Salmon (1984) developed an alternative account of explanation, which argued that explanations point to causes of phenomena, thereby articulating explanatory relevance not in terms of statistical relevance but causal relevance. This underscores again the difference between prediction and explanation: all correlations can be used for the purposes of prediction, but correlation need not imply causation, as required for causal explanation. It is not surprising that explanations in science are often causal explanations. Yet this had not been of relevance to philosophers working with a logical positivist framework such as Hempel, who addressed epistemological issues but did not commit themselves to metaphysical views such as the existence of a mind-independent reality and a particular causal structure of the world. In contrast, Salmon developed his account of causal explanation based on an analysis of causation as a physical process. Made explicit by later accounts, exhibiting causes is explanatorily relevant as causes are difference makers: the effect is more likely given the presence of one of its causes than this cause's absence (Woodward 2003). Even though the deductive-nomological model proved to be problematic, laws of nature can play a role in scientific explanations. This has most

\footnotetext{
${ }^{1}$ Brigandt (2010c) presents a special notion of inference according to which explanations are inferences. However, this is not to deny the differences between predictions (arguments) and explanations, but to argue that what makes something a prediction or an explanation does not depend on its logical structure, but on the specific (empirical) content involved.
} 
ably been captured by Philip Kitcher’s (1989) account of explanation, which ties explanation to the unification of knowledge. On this approach, an explanation is not any logical deduction from a law or law-like generality (as in the DN model), but a deduction occurring within the most unified system of our overall knowledge. The 'John and the birth control pills' deduction is not an explanation on Kitcher's unification account, as there is a more unified deduction that entails that (and explains why) no man gets pregnant.

As a result, the criticism of Hempel's model of explanation resulted in quite different accounts of what a scientific explanation consists in being proposed and endorsed by philosophers of science. Some (including Salmon) acknowledged that no single account captures all instances and aspects of explanation in science. This is especially true in philosophy of biology, where many scholars maintain that different kinds of explanations can be found across biology (Brigandt 2011c). In evolutionary biology and ecology, individual phenomena are often explained as being instances of quantitative generalizations. Mathematical models in these fields typically represent the dynamics of biological systems, such as change in gene frequencies, the size and structure of populations, or the geographical distribution of several species. The main mathematical basis of evolutionary biology is provided by population genetics, quantitative genetics, and molecular evolutionary genetics. There is not necessarily a single overarching mathematical theory for evolutionary biology; rather, evolutionary genetics is best viewed as consisting of a set of overlapping mathematical models (Lloyd 1994). Among the diversity of specific models, a unifying theme is that evolutionary change results only if there is heritable variation among entities and natural selection leads to the differential propagation of these entities. Yet different kinds of entities (genes, phenotypic traits, groups of organisms) can be subject to selection and undergo evolutionary change, and selection can occur on several levels at the same time (Okasha 2006), so that most models attempt to capture only some aspects of the 
evolutionary process, such as the migration of individual organisms within a few populations of one species, which need not directly feed into the unifying theme of change by heritable variation and selection. To a large extent, evolutionary theory is a statistical theory. This results from the fact that populations are always finite and can be quite small, so that random sampling effects occur, so-called genetic drift (Beatty 1984; Millstein 2002; Walsh et al. 2002). Probabilities can be assigned to different evolutionary trajectories and outcomes, and some evolutionary explanations are statistical explanations in that they explain a certain outcome as being more likely given the actual initial and boundary conditions (e.g., fitness differentials due to a certain selection pressure) than given some alternative conditions (Sober 2008). The probabilistic nature of evolution (captured by statistical models) is quite important, as random genetic drift insures that a population (generally moving up a fitness differential due to selection) need not get stuck with a phenotype whose fitness is only locally optimal, but can explore other, initially less optimal phenotypes so as to arrive at a more global fitness maximum (Brandon and Carson 1996).

In such fields of biology as physiology, developmental biology, and molecular biology (which may be referred to by the label 'experimental biology', Weber 2005), causal explanations are widespread. These fields of biology are to a substantial part concerned with the discovery of mechanisms, and phenomena are explained in a causal-mechanistic fashion. While Salmon’s account of causal explanation appealed to causation as a property of processes described by physics, philosophers of biology have come to make recourse to the notion of a mechanism to capture causal explanation in experimental biology (Bechtel 2006, 2008; Craver 2007; Darden 2006). A mechanism includes several entities, often different kinds of entities, and their causal interactions, such as mechanical or chemical interactions (Machamer et al. 2000). A mechanism is temporally extended, where some of the entities involved change their positions or their 
properties as a result of the mechanism's actions. A mechanism is a feature in the natural world; and what offers the mechanistic explanation is a scientific representation of relevant aspects of a mechanism. What is explained is the outcome state of a token mechanism, or the kind of behavior that is regularly produced by a type of mechanism. For instance, neurotransmission occurs in many different types of neurons in an individual and in different species, yet it involves a basic type of mechanism: the initiation and propagation of an axon potential along an axon’s membrane. An important aspect of discovering mechanisms and explaining in terms of mechanisms is to decompose a whole system into explanatorily relevant parts (Bechtel and Richardson 1993; Winther 2011), and to understand both the specific spatial and temporal organization of a mechanism that enables it to produce the phenomenon to be explained. While a mathematical model in biology explains by showing how the dynamic behavior or outcome of a process follows from certain (ideally simple) equations, a causal-mechanistic account explains by showing how the behavior or capacity of a complex system results from the interaction of its parts, where none of the parts may have the complex capacity. For example, while long-term memory is not a property of an individual neuron or an individual neuronal circuit, biologists might seek a mechanistic explanation of long-term memory in humans in terms of physiological changes in the states of various neurons.

So far I have emphasized that there are different kinds of explanations in biology, by pointing to the importance of mathematical models and statistical explanations in evolutionary biology and ecology, and the prevalence of causal-mechanistic explanations in the different fields of experimental biology. But this is not to say that a biological field uses only one type of explanation. Causal explanations are important in evolutionary biology and ecology as well. For beyond the generality of mathematical models covering many cases, an explanation of a specific case must make plain how a mathematical model applies to a concrete biological situation, 
including what causal aspects of it lead to the dynamics captured by the equations. A mathematical model from population genetics may predict an increase in the prevalence of a phenotype given that its fitness is larger than the fitness of other phenotypes. An application to a concrete situation has to causally explain why their specific phenotypic traits endow some organisms with a reproductive success (fitness) higher than that of organisms possessing other traits, based on the organisms’ actual environmental conditions, i.e., a causal explanation of why natural selection favors some phenotypic traits over others in this context. Likewise, quantitative models can be found in experimental biology, especially in the field of systems biology. In developmental biology, some specific developmental systems have been mathematically modeled so as to describe the dynamics of gene regulation within gene regulatory networks, or the formation of morphological patterns based on cellular interactions (on the development and evolutionary origin of the vertebrate limb skeleton, see Newman and Müller 2005). While explaining in terms of mathematical models and in terms of causal mechanisms have occasionally been described as distinct and potentially conflicting ways of biological theorizing (Winther 2006), both modes of explanation are sometimes jointly used by research groups, e.g., an ecological explanation in terms of theoretical models applied to the concrete causal interactions of ecological communities, which were obtained from field studies.

In line with the practice of biology, many philosophers of biology endorse a 'pluralism' about explanation, but we have to be precise about what this involves. It is a pluralism about the character of scientific explanation, in that two explanations (of two different biological phenomena) can be of a different kind — a mathematical-statistical explanation may be given for one phenomenon and a causal-mechanistic explanation for another phenomenon. A significantly stronger pluralism about explanation would maintain that one and the same phenomenon can be explained differently. For most philosophers this is an untenable position if the two explanations 
are somehow incompatible, though it is less controversial if two explanations of a phenomenon differ but are compatible, e.g., by each focusing on different causes involved in the production of the phenomenon to be explained (I shall return to this in Section 5). In any case, given a widespread pluralism about the character of scientific explanation, philosophers of biology are not concerned with arguing which model of explanation (statistical or causal) is the right one; rather, the philosophical task is to articulate in concrete biological cases which kind of explanation it is and what makes the empirical account explanatory.

This has implications for science pedagogy. Ideally, science education should faithfully present science in all its diversity. This includes the fact there are different kinds of explanation used across biology, so that teachers should avoid conveying a misleading picture about science by presenting only a limited or biased set of biological explanations to their class. However, the enormous complexity of contemporary biology raises serious challenges, both in terms of what science teachers would have to know about current biology and how to convey this to students in a suitable fashion. The biological explanations presented have to be accessible and compelling to students, which may not be the case for mathematical explanations. At the same time, conveying that biology is characterized by a plurality of kinds of explanation would be an important lesson for students, as it is not just about different scientific facts and theories (the content of science), but about the very nature of science. Biology education is structured by domains (cell biology, evolutionary theory, etc.), but since different types of explanation can often be found in one biological domain, it should be feasible to present distinct explanations in one part of a science class. It may also be worthwhile to portray the interaction of mathematical modeling and experimental / field work, as apart from being faithful to actual scientific practice this helps to see students the connection among different methods and ideas, as opposed to presenting to them somewhat isolated scientific issues. 


\section{Reduction and Biological Practice}

One special kind of explanation is reductive explanation, for example, explaining a higher-level phenomenon such as human brain development in purely molecular terms. More generally, reduction pertains to the structure of scientific knowledge. How is the knowledge from different scientific fields related? Reductionism often maintains that the knowledge from one scientific domain (typically at higher levels of organization) can be explained by the knowledge of another field (typically at lower levels of organization). A strongly unified vision of science could claim that there is a fundamental theory (e.g., fundamental physics) to which all scientific knowledge can be reduced. The received model of reduction, called theory reduction, was developed by Ernest Nagel (1949, 1961), using a logical positivist framework that characterized a theory as a set of statements in a formal language, including law statements. Theory reduction obtains when all of a theory (the so-called reduced theory) or at least the theory's laws can be logically deduced from another theory (the so-called reducing theory). In the late 1960s, inspired by the progress in early molecular biology, Ken Schaffner $(1969,1976)$ applied the model of theory reduction to biology, claiming that classical genetics was in the process of being reduced to molecular genetics and biochemistry. The ensuing reductionism debate in the philosophy of biology primarily centered on whether classical genetics was reduced to, or rather replaced by, the new molecular genetics (Hull 1974; Ruse 1971).

Schaffner's theory reduction model was immediately criticized and has been widely rejected (for more details see Brigandt and Love 2008). Critical reactions have taken two—not necessarily incompatible_-forms. The first response was the acknowledgement that some sort of reduction takes place in experimental biology, while insisting that the model of theory reduction fails to capture it. This was often accompanied by attempts to develop alternative accounts of 
what reduction is. The second response was more anti-reductionist in spirit, often concluding with an endorsement of pluralism, i.e., the idea that even future biology will consist of different fields, theoretical approaches, and explanations. Both reactions proceeded from the fact that the proponents of theory reduction could defend only the in principle possibility of reduction, whereas the critics wondered how this contributed to philosophically understanding actual biological practice, including reductionistic methods and reductive explanations (Wimsatt 1979). As mentioned in the introduction, the logical positivist dichotomy between the context of discovery and the context of justification has been rejected especially in the philosophy of biology. Discovery is not a non-rational process; molecular biologists have good scientific reasons for carrying out experimental and investigative steps based on prior knowledge (Hacking 1983; Weber 2005, Ch.3). The reductionism debate in biology illustrates the recent philosophical focus on scientific practice, including the close connection between the discovery of molecular mechanisms and explanations in terms of mechanisms.

Theory reduction presupposes that the concepts of the theory to be reduced are related to the concepts of the reducing theory — 'allele' (the notion of the gene in classical genetics) is not a term occurring in biochemistry, so alleles have to be related to biochemical phenomena before a reduction of the theory of classical genetics can proceed. Criticisms of theory reduction typically proceeded from the fact that the kinds of a higher level field and a lower level field stand in many-many relations, so that no systematic correspondence between the concepts of the two theories can be obtained (Hull 1974, Ch.1; Kitcher 1984). First, what is one natural kind or one unified phenomenon from the point of view of a higher level field can be different kinds or types of phenomena for a lower level field. A phenotypic difference, which is due to a difference in one allele according to classical genetics, can by the lights of molecular genetics be brought about by causes that are different in kind and not connected to one another other: a point 
mutation within a molecular gene's coding region, a mutation in regulatory region preceding the gene, or a mutation elsewhere in the genome. A morphological structure that is recognized as the same structure in different species can develop by means of quite different developmental processes and the involvement of different genes in these species (Brigandt and Griffiths 2007; Laubichler and Wagner 2001). As a result, an explanation in terms of the concepts of a higher level discipline can be more unified (encompassing many instances, possibly different species) and addresses the relevant natural kinds / categorizations underlying the phenomenon to be explained, while describing the situation in terms of a lower level science would require different accounts for some instances, where these accounts are not systematically related. (This is analogous to the multiple realization arguments against reduction known from the philosophy of mind; see Fodor 1974.) Likewise, a higher level feature can be causally more salient or more robust than a molecular feature, in that the higher level feature is much less affected by certain disturbances when it operates as a cause. In the case of genetic redundancy, the activity of a molecular gene is part of an overall mechanism producing a phenotype, yet experimentally deactivating the gene may lead to the same phenotype since other genes become activated to compensate for the defective gene. In this case, a whole gene network or developmental process is causally more robust than the single gene, so that explanations are more informative when they make reference to such robust higher level causes.

Second, a lower level kind or phenomenon can correspond to different higher level kinds or phenomena. This is because the effect of molecular entities and processes depends on and may vary with the context in which they occur. Wnt genes, coding for signaling proteins of the Wnt family, have many different functions in different species, including the control of muscle formation, blood stem cell formation, limb development (all in mammals), segment boundaries, eye development, wing development, and axon guidance (in insects). In one and the same 
organism, a gene can have many different functions in different cell and tissue types, e.g., wht1 is involved in mouse muscle formation and T-cell thymic proliferation, among other things. Consequently, non-reductionists have emphasized that the specific biological organization in which molecular processes are situated has to be studied (Hüttemann and Love in press; Laubichler and Wagner 2001). A reductionist may claim that the respective context of molecular entities can be taken into account, however, for the model of theory reduction (which aims at deducing a higher level theory from a purely molecular theory) this would require translating the total organismal context into molecular terms (Robert 2004, Ch.1). Therefore, at best the in principle possibility of theory reduction can be maintained.

As a result, several philosophers of biology have abandoned theory reduction and attempted to develop models of reduction that better capture actual explanatory practice in biology. Such accounts may be grouped under the label of explanatory reduction, as they intend to capture the nature of reductive explanations (Sarkar 1998; Weber 2005, Ch.2; Wimsatt 1976). While theory reduction assumes that what is reduced is a whole theory, a reductive explanation may account for a generalization of small scope or even a single phenomenon. Rather than explaining in terms of (a fundamental theory’s) laws, explanations in molecular biology are causal explanations, typically in terms of molecular mechanisms (Section 2). While the approach of theory reduction—originally developed within the context of logical positivism—is free of any ontological commitments (reduction is conceived as a logical derivation among linguistic statements), many accounts of explanatory reduction explicitly capture that reductive explanations in experimental biology proceed by explaining a whole in terms of its lower level parts, so that part-whole relations and ontological hierarchies are crucial. Most importantly, molecular explanations are articulated in a piecemeal fashion. Such explanations may focus on particular molecular causes, which in a certain organizational context bring about a specific 
higher level effect. Biologists can take this organized context for granted and need not reduce it to the molecular level, and still provide a causal explanation—as it points to one of the various molecular factors involved in the production of the phenomenon to be explained. In opposition to the focus on finished science promoted by in principle theory reduction, explanations in molecular biology never capture all of the causal factors involved. In actual practice, molecular explanations are set up in a stepwise fashion; explanation in terms of mechanisms is closely related to the piecemeal discovery of such mechanisms. Causal-mechanistic explanations are often based on partially known mechanisms - in contrast to many textbook presentations, scientific knowledge is always in the process of development and modification. Rather than viewing biology as solely consisting of theories exhibiting unchanging logical relations, a philosophy of biology that is concerned with articulating the epistemology of scientific practice and explanation as it occurs in actual biology has to pay attention to the transformation of individual explanations and of the structure of knowledge during the process of discovery.

The most common response among philosophers of biology to the failure of the theory reduction model and its monolithic vision of the structure of scientific knowledge has been an anti-reductionist stance, typically in the form of an endorsement of pluralism (Mitchell 2003). Given that reduction to one fundamental field (e.g., molecular biology) is impossible, actual biology consists in a diversity of fields and theoretical approaches. This is a more general pluralism than the pluralism about the character of explanation discussed in Section 2 (which maintained that different kinds of explanations exist across biology) because it asserts that biology is characterized by and in fact needs a plurality of methods, theories, and explanatory perspectives, as opposed to a unique and overarching approach. Even one biological field requires many concepts, methods, modes of explanation and explanatory approaches. Occasionally, pluralists have maintained the much more contentious position of the disunity of 
biology (Dupré 1993; Rosenberg 1994).

The fact that modern biology is not exclusively reductionistic also matters for science education. One reason I want to highlight is the acceptance of evolutionary theory among high school students, which in some countries is threatened by religiously motivated objections (which nowadays often go by the label intelligent design 'theory'). One motivation for skepticism about evolutionary theory is the assumption that evolutionary biology (and science in general) paints a materialistic and reductionistic picture of life and the natural world. Actually, in their development and life-time organisms can react in a surprisingly flexible fashion to various disturbances. This includes dealing with the influence of a constantly changing environment, but the above example of genetic redundancy shows that even genetic changes (including the experimental deactivation of a gene deemed to be developmentally important) can be largely compensated for by the organismal system, so that the genetic change does not result in a phenotype with lower survival ability. In recent evolutionary developmental biology this has been deemed to be the basis of evolvability, which is the ability of organisms to generate novel morphological variation that is viable and functional, so that fast morphological change in evolution can occur (more on evolvability in Section 5). Whereas explaining the ability of morphological evolution requires viewing organisms as flexible systems (Kirschner and Gerhart 2005), intelligent design proponents erroneously portray organisms as complex machines, so as to prime the implication that these machines were designed by someone. Michael Behe’s (1996) irreducible complexity argument against evolution claims that organismal systems consist of parts that are arranged in a functional manner, but where the removal of any part leads to a breakdown of the system's function. The irony is that it is not actual biology who endorses a reductionistic picture of organisms, but intelligent design by claiming organisms to be rather inflexible machines — an issue high school students should be made aware of. But if modern 
biology is not exclusively reductionistic, we need an alternative vision of biology that can be conveyed to students, to which I turn in the next section.

\section{Explanatory Integration}

A drawback of using the label 'anti-reductionism' as part of standard critiques of reduction is that it suggests that any approach that cannot be reduced (e.g., classical genetics) is a completely autonomous field that does not have any connections to other fields. Yet both the classical gene concept and the molecular gene concept are used in tandem by many contemporary geneticists (Vance 1996; Weber 2005, Ch.7). In the philosophy of mind, most debates about reduction have been framed in terms of two seemingly separate levels - the mental level and the physical level—with the question being whether and how mental properties and processes can be reduced to physical properties and processes. Yet such a two-level scheme ignores the way in which different disciplines are actually involved (neuroanatomy, neurophysiology, developmental neurobiology, cognitive neuropsychology, cognitive psychology), studying entities on several levels and relating various aspects of cognitive structure and function (long-term potentiation of individual neurons, impact of brain lesions on specific cognitive functions, perception, cognition involving concepts). A two-level scheme is particularly inadequate for modern experimental biology. Most philosophers endorsing ‘anti-reductionism' and 'pluralism' have not claimed that there are no connections among different theories or disciplines. But merely criticizing reductionist arguments does not yield a positive philosophical account of how different fields are related. Beyond the traditional dichotomy between reduction and anti-reduction, the most promising philosophical approaches are those that offer an epistemological study of how interdisciplinary research across different biological fields and the integration of disciplines 
proceeds, including factors that promote or limit integration, and the nature of explanations that involve knowledge from several biological disciplines (Bechtel and Hamilton 2007; Grantham 2004; Mitchell 2002). Although such explanations often appeal to molecular causes, they explain in terms of entities on several levels of organismal organization, so that using the label 'reductive explanation’ can be misleading.

The classical model of integration (dubbed 'non-reductive unification') was put forward by Lindley Darden and Nancy Maull (Darden and Maull 1977; Maull 1977). Their core idea is the notion of an interfield theory, which is a new theory that upon its establishment comes to connect two previously unrelated fields. One of their examples is the way in which the chromosome theory of inheritance came to bridge Mendelian genetics and cytology. In the early 1900s, Mendelian genetics attempted to understand patterns of phenotypic inheritance. Genes (alleles) were postulated for this purpose, but no claim about the material nature of genes was madeMendelian genetics was not concerned with the internal constitution of organisms, but with transgenerational phenotypic inheritance. Cytology (now called cell biology) dealt with the material contents and structures of cells, and thus (originally) had no connection with Mendelian genetics. The chromosome theory of inheritance was developed in the 1910s and stated that chromosomes as particular cellular structures are the material bearers of inheritance, and that genes are parts of chromosomes. This created a highly influential connection among the previously unrelated fields of Mendelian genetics and cytology. Note that this integration was not reductive: neither has genetics been reduced to cell biology, nor has cell biology been reduced to genetics. (The chromosome theory as an interfield theory did not reduce both genetics and cytology, either.)

A limitation of the interfield theory model is that it focuses on theories as the units of 
analysis, and it has become clear that other notions apart from 'theory' are needed to capture the structure of biological knowledge. Consequently, recent accounts of integration in experimental biology have fruitfully employed the notion of mechanisms. Darden (2005) analyzes the relation between classical and molecular genetics in these terms. On her account, classical genetics focuses on meiosis as a special period of the cycle of germ-line cells (gamete formation), where the entities in the mechanism relevant for explaining patterns of inheritance are the chromosomes (segregating independently into different gametes) and larger parts of chromosomes (in the previous process of crossing-over). Molecular genetics addresses gene expression, and thus a different mechanism in that it concerns a different temporal period of the cell cycle and takes place in all cell types. The explanatorily crucial entities are below the level of chromosomes, including DNA segments, promoters, start and stop codons, regulatory sequences, and various other molecules in the nucleus or cytoplasm involved in gene regulation, transcription, and translation. The explanations of classical genetics do not reduce to the explanations of molecular genetics, as both address different mechanisms at different temporal periods. At the same time, the philosophical analysis in terms of mechanisms explains how the explanations of classical and molecular genetics are related, by detailing how gene expression and meiosis are different periods of an overall cellular process and how entities on different levels are related by standing in part-whole relations or interacting with each other (e.g., DNA segments are specific parts of chromosomes, histone structure influences the possibility of DNA transcription, linear DNA structure is involved in the process of chromosomal crossing-over). Such a mechanism-based account is particularly fruitful for the purpose of science education. The high school instruction on genetics I received presented classical genetics too much as a mathematical account (Mendel's law and how to calculate genotype ratios) and then went on to molecular genetics without sufficiently making clear how both were related-given that both involve genes and their 
effects. An account in terms of mechanisms can make the connection obvious to students.

Carl Craver (2005, 2007) uses the mechanisms framework to articulate multifield integration within neuroscience, emphasizing how mechanistic explanations relate entities on different levels. His primary example is the study of learning and memory, and its relation to the cellularmolecular process of long-term potentiation (synaptic plasticity). The latter is clearly implicated in memory formation, yet long-term potentiation is not just a molecular counterpart of memory and there is no structural-functional correspondence between the two (that would make talk about one being reducible to the other legitimate). Rather, long-term potentiation is a lower-level component of an overall, multilevel mechanism (memory formation), whose nature has yet to be adequately explained. Craver also points out that accounts focusing on reduction (and thus addressing relations among different levels) have overlooked the prevalence of integration among approaches addressing the same level, such as the fruitful interaction of neuroanatomy (studying the structure of neuronal circuits) and electrophysiology (studying the function of neuronal connections). His most important point is the need to take into account the historical dynamics of mechanistic research (see also Bechtel 2010). Accounts asserting reduction in neuroscience (e.g., Bickle 2003) have exclusively focused on finished reductive explanations, which ignores the methodological assumptions and explanatory agendas by which scientists actually discover those explanations. Craver's historical discussion shows that in addition to attempting to find the molecular correlate of a higher-level phenomenon (downward-looking episodes), there have also been attempts to understand the role of a molecular mechanism in a complex higher-level phenomenon (upward-looking periods of research). Progress in memory research has sometimes been made independently of or by abandoning reduction as an explanatory goal. Long-term potentiation was originally not discovered during a top-down search of the neural correlate of memory, but was noticed during neurophysiological studies 
(independently of research on memory). While in the 1950s and 60s long-term potentiation was initially seen as a kind of memory, subsequently it has been construed as a mere component of the overall mechanism of memory, which is to be understood by a multilevel analysis. This highlights again the close relation between discovery and explanation. Any philosophical framework which attempts to epistemologically account for scientific practice and how progress is actually achieved (rather than idealized and finished explanations only) has to take such nonreductive aspects of ongoing integrative endeavors into account.

It has repeatedly been observed that integration can be motivated by the existence of scientific problems that cannot be solved by any discipline in isolation. Alan Love (2005, 2008, this issue) goes beyond this by laying out how problems can structure integration, by foreshadowing how the contributions from several fields are to be coordinated and integrated in complex explanations (see also Brigandt 2010a). His core idea is the notion of a problem agenda and its associated criteria of explanatory adequacy. A problem agenda is a complex scientific problem, consisting of several component questions that must be addressed to produce an adequate explanation. A problem agenda is structured by systematic (e.g., hierarchical) relations among component questions, which indicate how answers to individual component questions relate to one another. Each component question (and thus the overall problem agenda) is tied to specific criteria of explanatory adequacy, which detail what would count as a satisfactory answer or explanation. Love's philosophical point is that given the particular criteria of explanatory adequacy associated with a certain problem agenda, the latter determines what biological fields are relevant to solving the problem, and what intellectual contributions from each field are needed. Moreover, due to its internal structure, a problem agenda foreshadows how the contributions from different fields are to be coordinated and integrated in the overall explanation. 
Love's example is the evolutionary origin of novelties. An evolutionary novelty is a morphological structure in a species that is qualitatively different from any structure of the species's ancestors. Examples of novelties are the evolutionary origin of bird feathers, and the origin of the vertebrate jaw, i.e., the transition from jawless fish (whose extant jawless descendants are hagfish and lampreys) to jawed fish. Explaining the evolutionary origin of novelty is a core problem for evolutionary biology, with currently major research efforts being devoted to various individual novelties (typically in the context of evolutionary developmental biology; Brigandt and Love 2010; Love this issue). Traditional neo-Darwinian evolutionary theory is ill-equipped to account for novelty. For its theoretical core population genetics studies the quantitative change in gene frequencies, based on natural selection favoring certain existing variants - which does not explain how qualitatively novel morphological variants originated in the first place. It is clear that the explanation of novelty requires contributions from many biological fields, including phylogeny, paleontology, developmental biology, and morphology. On Love's (2008) account, the explanation of the origin of novelty is a problem agenda, which determines how the various contributions from different disciplines are to be coordinated (see also Brigandt 2010a). For example, a first step is to lay out a sequence of relevant morphological traits in different species within a phylogenetic lineage, leading up to the presence of the novelty. For this component question, paleontology is needed to provide fossil data about the structures studied. The field of phylogeny is likewise relevant, as the proper evolutionary tree of the species involved entails at which phylogenetic junctures morphological changes took place. The second basic step (component question) in accounts of novelty is a causal-mechanistic explanation of how structural changes came about. Developmental biology is central here, as it has to be explained how ancestral developmental systems (organisms) were modified so as to result in a changed developmental system producing the qualitatively new structure (Müller and Wagner 
2003; Wagner 2000). Traits on several levels of organization are to be considered, given that the generation of such a structure as the vertebrate jaw is to be explained. The need to address the activities of genes, cells, and tissues across developmental time, and the changes in development across evolutionary time provides a template to coordinate the explanatory inputs from different disciplines.

In contrast to an argument against reductionism or a mere statement of pluralism, the problem agenda framework offers a positive epistemological account of how intellectual resources from different fields are related. Since problem agendas coordinate interdisciplinary research and structure explanatory integration, 'problem agenda' is an epistemological notion that is required in addition to traditional notions (such as theory, law, method, explanation, and mechanism). In science education, apart from the fact that contemporary biology is interdisciplinary, it is important to teach what drives interdisciplinary research. The idea of problem agendas yields further implications about the nature of science (Brigandt 2010a). Philip Kitcher (1999) acknowledges that a real unification in biology is impossible, given the complexity of the biological world. But he argues that unification is a regulative ideal, in that scientists should strive for as much unity as nature permits. In contrast, I point out that unification is not a universal aim of scientists. It is true that some integration is needed to solve certain biological problems. But some other problems can be solved without any additional integration; and moreover, a certain specialization into different disciplines can also be conducive to solving complex problems. Bill Bechtel $(1986,2006)$ has emphasized how the creation of new disciplines leads to both integration and specialization/disintegration. As a result, how much integration (and what kind of a combination of integration and specialization) is needed depends on and varies with the scientific problem under consideration. Contra Kitcher, integration/unification is not a regulative ideal or an aim in itself; instead, integration may be 
needed for the purpose of solving a certain problem.

A problem-focused epistemological framework also captures some dynamical aspects of the structure of science. A scientific discipline may address a problem, and subsequently come to establish integrative connections to other fields. At the same time, this discipline may also deal with another problem (or come to address it at a later historical point). This second problem is likely to require different items of knowledge to be integrated, possibly involving connections to a different set of fields than the first problem. Thus, a discipline entertains several sets of (overlapping) integrative relations among different items of knowledge and different disciplines, where the problem addressed in a certain research context determines which set of intellectual connections is operative in this context. This feature of scientific practice clashes with the picture advocated by some reductionists, who have envisaged a linear hierarchy of disciplinesmicrophysics on the lowest level, chemistry and molecular biology on higher levels, organismal biology and sociology on even higher levels-where a lower level theory can reduce the ones on higher levels (Oppenheim and Putnam 1958). Yet in contrast to this so-called layer-cake picture of science, there is no linear epistemological hierarchy of disciplines. Intellectual approaches exhibit complex relations, and there is no unique set of relations among scientific fields, as the relevant intellectual connections can vary from context to context, depending on which problem is considered (Brigandt 2010a). Such a philosophical focus on the practice and dynamic operation of science comports well with a pedagogy that teaches sciences as an inquiry, rather than merely as a set of established facts (Love this issue).

\section{Idealization and Explanatory Aims}

Mathematical models and other scientific representations often make idealizations by abstracting 
away from certain aspects of natural phenomena (Weisberg 2007; Wimsatt 2007). Idealizations make a situation represented less complex and permit quantitative models that are mathematically more tractable. Idealizations may leave out irrelevant detail while focusing on scientifically important aspects of a complex phenomenon. Idealizing and abstracting away can generate scientific representations that are more general in that they capture many concrete situations, despite the differences among them. For instance, for the sake of mathematical simplicity, some models in population genetics assume that populations consist of an infinite number of organisms, even though populations are finite, which creates particular effects on evolutionary change. Or a model may study evolutionary change at a few genetic loci, though organisms possess a plethora of genes that interact so as to influence evolutionary dynamics. Idealizations are by no means unique to mathematical models and statistical explanations, but are widespread in causal and mechanistic explanations. Out of a total set of causes bringing about a phenomenon, some causes have a greater influence than others, and a causal explanation may focus on some causes while relegating others to the background. An explanation in terms of mechanisms has to break down a complex whole into parts (and explain in terms of the causal properties and interaction of the parts). Any system can be decomposed into smaller units in different ways, but a mechanistic explanation usually chooses one decomposition only (Craver 2009; Winther 2006).

Making idealizations raises philosophical issues and the strategy of abstracting away from empirical details has been criticized. For instance, construals of biological species as natural kinds with essences focus on some features that are shared among the members of a species (essential properties), thereby inevitably ignoring within-species variation, which must not be neglected on any account of why and how species evolve (Sober 1980). However, any scientific model and representation serves particular scientific purposes, such as certain explanatory aims 
(Love 2009; Weisberg 2007). Relative to a concrete explanatory aim, the idealizations made by a representation or explanatory model can be scientifically legitimate if the model ignores only those empirical details that do not contribute to the explanatory aim. In fact, ignoring some while focusing on other, scientifically relevant features makes the model explanatory. At the same time, the abstractions made may limit the applicability of the model because particular idealizations may make the model useless or problematic relative to other explanatory aims (for which the model was not originally developed). This may hold for a model not being suitable for other empirical cases, or for the original case being approached with a novel explanatory aim. For example, descriptions in developmental biology break the development of a model organism species into normal stages (e.g., molt-to-molt intervals in insects). While this is useful for explaining development, the distinct explanatory aim of accounting for the evolution of development may require a different representational framework because developmental biology’s normal stages obscure natural variation in development, phenotypic plasticity, and the way in which developmental stages are created and transformed in evolution (Love 2009, 2010, this issue). Explaining the development of a species and explaining the evolution of this species’s development are two different explanatory aims about the same empirical object, and different representations of this object are needed to meet the respective aims. Scientific representations, models, and explanations are not all-purpose tools, but employed for specific scientific purposes and explanatory aims. This creates the philosophical need to study the explanatory aim underlying an explanatory model, the extent to which the idealizations made are legitimate, and whether a different explanatory model has to be used for other explanatory aims, which highlights that scientific explanations have to be studied together with the particular explanatory aims pursued by scientists.

To illustrate the relevance of explanatory aims in science by an example that is of 
importance to biology education (and primes further philosophical implications), let us consider the role of genes in explanations of development and phenotypic evolution. In explanations of development, it is clear that genes are only one among many causally relevant factors. The reason is that an explanation of development has to include an account of differentiation-the formation of different cell types, different tissues, and different organs within a developing organism, including the spatial and temporal pattern of differentiation and morphogenesis. Yet (nearly) all cells of an individual organism contain the same genome, so that reference to genes alone (or to genetic information) cannot possibly explain differentiation. What creates differences among cells and tissues is the way in which genes are differentially expressed in different parts of an organism. Gene expression is initiated and regulated by various non-genetic molecular factors inside the nucleus and the cytoplasm (transcription factors, proteins involved in signaling cascades, etc), and results in a specific spatio-temporal pattern due to cell-cell signaling and the organizational context of individual cells. As a result, explanations of development are epigenetic in that they essentially make reference to the interaction of molecular genes and various non-genetic factors, including entities on several levels of organismal organization. $^{2}$

When we turn to the explanation of phenotypic evolution, a more gene-centered framework seems to prevail. This stems from the fact that natural selection is a main cause of phenotypic change. Natural selection acts on phenotypic variation, but this leads to evolutionary change only

\footnotetext{
2 This is well-reflected by the contemporary notion of the gene in molecular biology, which takes into account the complex and diverse processes in which DNA segments lead to different gene products, where this gene activity is highly context-dependent and generated by non-genetic factors (Brigandt 2010b; Stotz 2006a, 2006b). Griffiths and Stotz (2007) call it the postgenomic molecular concept of the gene, as it stems from the postgenomic focus on genome wide gene function (beyond the mere study of the structure of individual DNA sequences).
} 
if the phenotypic variation is heritable (i.e., a phenotypic difference tends to reoccur in subsequent generations). Phenotypic variation can be due to all sorts of factors (e.g., environmental variations), yet heritable phenotypic variation is primarily due to genetic variation. ${ }^{3}$ Explanations in population genetics are in line with the classical gene concept, which conceives of genes as such factors that a genetic difference results in a phenotypic difference (Brigandt 2010b). This is not to say that a single gene causes the phenotype-the phenotype develops based on the interaction of many genes and the environment. But if two organisms just differ in one gene (other genes and the environment are identical and can be taken as part of the constant background), a phenotypic difference results. This suffices for explanations of phenotypic change due to natural selection, as selection acts on heritable phenotypic variation, so that the differences among individuals matter. As detailed above, an explanation of development makes non-genetic factors explanatorily essential. Yet a selection-based explanation of phenotypic evolution merely requires that genetic differences result in phenotypic differences (so that variation is heritable), and it is irrelevant how genetic differences developmentally lead to phenotypic differences (be it in a genetic or epigenetic fashion).

This statement of explanations of phenotypic evolution being gene-centered was too fast. For it holds only if the explanation appeals to natural selection, which is the case if the explanatory aim is the explanation of evolutionary adaptation. If we shift to another explanatory aim in the context of phenotypic evolution, the explanation of evolvability and/or the origin of evolutionary novelty, the picture changes (on evolutionary novelty see Section 4). Evolvability is

\footnotetext{
${ }^{3}$ Heritable phenotypic variation can also result from epigenetic inheritance and niche construction. Moreover, given that heritable phenotypic variation is sufficient for evolutionary change, one may wonder whether it matters for evolutionary explanations what the basis of heritable phenotypic variation is (Godfrey-Smith 2009). I ignore these complications here, as my focus is on contrasting explanations of adaptation with explanations of evolvability.
} 
the capacity of organisms to generate viable heritable phenotypic variation. Genetic variation is random, but it results in a non-random, highly structured phenotypic variation that is much more viable than if phenotypic variation was just random. An account of evolvability has to explain how viable phenotypic variation and rapid phenotypic change in very complex phenotypic traits is possible (Gerhart and Kirschner 2007; Kirschner and Gerhart 2005). This is due to the fact that the development of an organism somehow ensures that (a) a trait can vary independently of many other traits, so that selection can modify different parts of an organism independently of each other, and that (b) some traits of a complex character change together in a coordinated and integrated fashion, so that one genetic change can lead to a functional change in many phenotypic traits at the same time (Hendrikse et al. 2007). For this reason, development yields an explanation of evolvability, so that just like explanations of development, explanations of evolvability are not exclusively gene-centered, but epigenetic, i.e., in terms of the interaction of many genetic and non-genetic factors on several levels of organismal organization.

Adaptation and evolvability both pertain to phenotypic evolution; here is a way to conceive of their relation. There are two aspects to phenotypic evolution: (1) the generation of heritable phenotypic variation, and (2) the selection of phenotypic variation. An explanation of evolvability addresses the first aspect, attempting to understand how viable phenotypic variation is generated in the first place (while taking the presence of some natural selection merely as a background condition). An explanation of adaptation focuses on the second aspect, detailing what particular selection pressure obtains (while taking the presence of sufficient heritable phenotypic variation for granted without explaining it). Accounts of evolvability and adaptation feature an epigenetic framework (development) and a gene-centered framework (population genetics), respectively. The situation that one but not the other explanation pertaining to phenotypic evolution is gene-centered is legitimate, given that they address different explanatory 
questions. Explanations of adaptation and of evolvability address different aspects of the overall process of phenotypic evolution, focusing on some factors while ignoring others—abstracting away from the factors is warranted given the respective explanatory aim.

Both explanatory frameworks (of adaptation and evolvability) are compatible as they address different aspects of an overall phenomenon. However, this (in principle) compatibility is recognized only to the extent to which individual biologists are aware of the fact that there are different explanatory aims pertaining to phenotypic evolution, and agree that these explanatory aims are legitimate and that different explanatory frameworks are needed for these different aims. Evolution is neither primarily explained by population genetics nor primarily by development. Many biologists are aware of this, but some still prefer their individual explanatory approaches. The same applies to the problem-based philosophical account of explanatory integration, laid out in Section 4 in the context of explanations of the evolution of novelty. There the epistemological point was that a problem agenda-due to its internal structure and the associated criteria of explanatory adequacy—determines how the explanatory contributions from different biological disciplines are to be coordinated. To be sure, this results in actual integration only if the biologists from the different fields involved agree on the basic aspects of the joint complex problem agenda and the criteria of explanatory adequacy. Consequently, it is vital for philosophers to take into account the particular explanatory aims underlying individual explanations developed and debated by scientists. For explanatory aims determine whether different explanations are actually competing or complementary and why conflict among scientists results.

Given that the same natural phenomenon can be approached by different explanatory 
projects, different explanatory frameworks about a phenomenon can legitimately coexist. ${ }^{4}$ As argued in the previous section, an integration of different frameworks has to occur only if it is scientifically required (e.g., for the purpose of solving a complex problem). The impact of explanatory aims opens the possibility of a much stronger kind of pluralism than the varieties of pluralism mentioned so far, which have highlighted that different types of explanations are used for different empirical cases, or different explanatory frameworks are used for one overall phenomenon - though these explanations are complementary and can in principle be integrated. Nancy Cartwright (1983) has argued that while models that attempt to truly represent as many features of a phenomenon as possible are hardly explanatory, basic theories obtain explanatory purchase precisely by focusing on some idealized laws or causal capacities (even though these idealized theories never directly represent concrete situations). An idealization need not consist in merely leaving out some features of reality—so that these features could later on be added to the account—but the idealization may actually distort some aspects reality and still be explanatory, precisely because these idealizations are made. If one overall phenomenon is approached with different explanatory aims, then different explanatory models may be put forward that make use of distorting idealizations that are mutually incompatible, and thus cannot be integrated into one model that more completely represents nature (Kellert et al. 2006). This is a more contentious version of pluralism, as it maintains that different explanations of a phenomenon may coexist that cannot possibly be integrated. Whether and when this type of pluralism obtains depends on the concrete empirical case and the representational and explanatory ideals used by scientists (Odenbaugh 2003; Weisberg 2006, 2007). In any case, the lesson for science education is this. Idealized models are very successfully used for the purposes

\footnotetext{
${ }^{4}$ For a discussion of explanations focused on form and explanations focused on function in evolutionary biology, see Love (this issue).
} 
of teaching, as they direct students’ attention to scientifically important features. But apart from ensuring that a given scientific content is effectively presented to students by the use of an idealized model, it is important to remind them that at the same time an explanatory model can obscure some features of nature-features that can be important relative to other explanatory aims. Scientific models are not all-purpose tools, but useful only for certain explanatory aims, and alternative models may be needed in an empirical domain. ${ }^{5}$

It deserves emphasis that explanatory aims operate on a different level than explanations and other scientific representations. Explanations, models, descriptions of mechanisms, theories, and scientific beliefs are all representations of the phenomena studied by science. Yet explanatory aims are not about the phenomena studied by science, they are about science itself. Explanatory aims are values of scientists — a feature clearly not taken into account by the deductivenomological model of explanation, which focuses on logical relations among formal statements. ${ }^{6}$

\footnotetext{
${ }^{5}$ Explanatory aims are relevant even in philosophical studies of natural kinds, which have usually been approached from a purely metaphysical point of view. A natural kind is a grouping of objects that is not just a matter of human convention, but reflects the structure of nature. The standard project is to develop a metaphysical construal of what a natural kind is, and what distinguishes natural kinds from other kinds. However, I contend that epistemological issues are equally important, in particular the philosophical study of which particular explanatory aims scientists pursue when using kinds in their theorizing, and whether and how a grouping of objects into a kind meets the given explanatory aim (Brigandt 2009, 2011b).

${ }^{6}$ The philosophical importance of values in science has been particularly stressed by social studies of science and feminist philosophy of science (Douglas 2009; Kourany 2010). While some philosophers may still aim at a clear separation of epistemic and other values (where only epistemic, but not personal, socio-political, and economic values are a proper part of science and a concern for philosophy of science), at least in current biomedical research various values are so strongly intertwined in the production of knowledge that in my view a distinction between epistemic and non-epistemic values is not philosophically fruitful. A philosophy of science that endeavors to study which kinds of scientific research is socially responsible will insist on the relevance of socio-political values from the outset (Kourany 2010). While it is beyond the scope of this essay's topic, I view values in science to be of importance for science education as well. Science ought to be taught more as a social process that is based on institutional factors and various interests. Understanding debates about global warming and evolution not only
} 
It is precisely for operating on a different level than representations that explanatory aims are of particular philosophical importance, as they account for the dynamics of science (Brigandt 2010b, 2011b, forthcoming). Explanatory aims guide scientific discovery and attempts at developing explanations. Explanatory agendas contribute to the intellectual identity of scientific approaches, and the distinct explanatory aims of different biological fields account for the diversity within biology and the presence of different trends in different parts of biology. The existence of different explanatory aims can hamper communication across different disciplines, and result in conflict when the same phenomenon is approached with different explanatory aims by different scientists. At the same time, the recognition of an explanatory aim shared by different fields can motivate and coordinate interdisciplinary research. Apart from influencing the dynamic behavior of science by guiding the development of explanations and motivating changes in various scientific representations (in the attempt to improve scientific accounts vis à vis the explanatory aims), the explanatory aims pursued can be subject to change themselves, further enhancing the transformation of scientific knowledge. For a philosophy of biology that aims to capture scientific practice and the workings of science, this is a compelling reason to take explanatory aims into account.

\section{Conclusion: Lessons for Biology Teaching}

This essay has analyzed and developed views about explanation in contemporary philosophy of biology. Philosophers have parted with the Hempel-Oppenheim model of explanation (derivation 
from laws) by endorsing the existence of different kinds of explanations, including statistical and causal-mechanistic explanations. The lesson for the teaching of biology is to convey to students the diversity of kinds of explanations and the heterogeneity of modes of reasoning found in contemporary biology. Apart from a mere diversification of models of explanation, the philosophically most interesting developments of the last few decades are those that tie explanation to a variety of other aspects of scientific theorizing and practice, going beyond the logical positivist view of explanation as a logical relation among formal statements by studying explanation as inseparable from overall scientific activity. Especially in the context of mechanistic explanations in experimental biology, explanation is intimately tied to discovery, which has resulted in the rejection of accounts of theory reduction and the development of models of explanatory reduction.

Biology is only partially reductionistic, and mechanistic explanations appeal to the interaction on several levels of explanations. Of particular philosophical importance is interdisciplinary research and complex explanations that use contributions from different biological disciplines, and my discussion laid out an epistemological account of explanatory integration that views integration as being coordinated by problem agendas. Moreover, explanations (as representation of the natural world) cannot be philosophically separated from explanatory aims (as aims of scientific activity). Explanatory aims determine whether different explanations are competing or complementary, and to which extent idealizations made by explanatory models are legitimate. Specific explanatory aims are a major determinant of the microstructure and dynamics of scientific activity—especially when different groups of scientists pursue different explanatory aims, or when explanatory aims change across time. Generally, rather than offering definitions of what an explanation is, philosophers of biology have come to examine how various explanations operate in the dynamic process of the generation and 
employment of scientific knowledge. The implication for science education to portray biology as an interdisciplinary practice, where scientific problems and explanatory aims motivate and guide research. It is useful to remind students that given that idealizations are made (which can obscure some features of nature), explanatory models are not all-purpose tools, but valid only relative to particular explanatory aims. Alternative models may exist for a phenomenon.

I have distinguished different kinds of pluralism. The first is a pluralism about the character of explanation, asserting that different explanations may be of a different type (e.g., mathematical vs. causal-mechanistic). Another type of pluralism is broader, maintaining that biology needs a diversity of methods, concepts, explanatory frameworks, and theories, where one explanatory framework cannot be reduced to another one. Both admit the possibility that different kinds of explanations are needed for different phenomena, but a very different kind of pluralism explicitly asserts that different explanatory frameworks may be used for a single phenomenon, because there are different legitimate explanatory aims and questions that different scientists can pursue in the study of the phenomenon. Sometimes different such explanatory framework are complementary, but if it is the case that several explanatory models pertaining to this phenomenon cannot be integrated as they make jointly incompatible idealizations (necessitated by different explanatory aims), a strong pluralism results that allows for the coexistence of explanatory models that make incompatible claims about a phenomenon.

In line with the recent philosophical focus on scientific practice, the general thrust of my recommendations for science education has been to present biology as a dynamic, inquiry-based practice. This presents concrete pedagogical challenges. Given the diversity and complexity of contemporary biology, is it demanding for teachers to know about the basic shape of current biological theories. Moreover, any move from solely teaching the content of biological theories 
to also teaching biology as a practice requires going beyond the selection and presentation of various suitable scientific explanations_-which are lessons about nature-and to convey to students why biologists come to form certain explanations and how they use them- which are lessons about the operation of science. But it is worthwhile to tackle this pedagogical challenge. It is impossible for teachers to keep up with the latest scientific development, so rather than attempting to give students an idea of recent important theories, it may be more productive to convey to them how scientists come to confirm theories and to develop new theoretical perspectives. Most importantly, the nature of science is to constantly revise knowledge, so that teaching how science as an investigative practice works is crucial for making students understand how scientific knowledge is modified and why and how new explanations are generated. There is a high educational value in making students aware of and reflect on the nature, limits, and aims of science in general, and of individual scientific explanations.

\section{Acknowledgements}

I am indebted to Kostas Kampourakis, Alan Love, and an anonymous referee for comments on an earlier version of this essay. This work was funded by the Social Sciences and Humanities Research Council of Canada (Standard Research Grant 410-2008-0400). 


\section{References}

Beatty, J. (1984). Chance and natural selection. Philosophy of Science, 51, 183-211.

Bechtel, W. (1986). Integrating sciences by creating new disciplines: The case of cell biology. Biology and Philosophy, 8, 277-299.

Bechtel, W. (2006). Discovering cell mechanisms: The creation of modern cell biology. Cambridge: Cambridge University Press.

Bechtel, W. (2008). Mental mechanisms: Philosophical perspectives on cognitive neuroscience. London: Routledge.

Bechtel, W. (2010). The downs and ups of mechanistic research: Circadian rhythm research as an exemplar. Erkenntnis, 73, 313-328.

Bechtel, W., \& Hamilton, A. (2007). Reduction, integration, and the unity of science: Natural, behavioral, and social sciences and the humanities. In T. A. F. Kuipers (Ed.), General philosophy of science: Focal issues (pp. 377-430). New York: Elsevier.

Bechtel, W., \& Richardson, R. C. (1993). Discovering complexity: Decomposition and localization as strategies in scientific research. Princeton: Princeton University Press.

Behe, M. J. (1996). Darwin's black box: The biochemical challenge to evolution. New York: Free Press.

Bickle, J. (2003). Philosophy of neuroscience: A ruthlessly reductive approach. Dordrecht: Kluwer.

Brandon, R. N., \& Carson, S. (1996). The indeterministic character of evolutionary theory: No no hidden variables proof but no room for determinism either. Philosophy of Science, 63, 315-337. 
Brigandt, I. (2009). Natural kinds in evolution and systematics: Metaphysical and epistemological considerations. Acta Biotheoretica, 57, 77-97.

Brigandt, I. (2010a). Beyond reduction and pluralism: Toward an epistemology of explanatory integration in biology. Erkenntnis, 73, 295-311.

Brigandt, I. (2010b). The epistemic goal of a concept: Accounting for the rationality of semantic change and variation. Synthese, 177, 19-40.

Brigandt, I. (2010c). Scientific reasoning is material inference: Combining confirmation, discovery, and explanation. International Studies in the Philosophy of Science, 24, 31-43.

Brigandt, I. (2011a). Critical notice of Evidence and evolution: The logic behind the science by Elliott Sober, Cambridge University Press, 2008. Canadian Journal of Philosophy, 41, 159186.

Brigandt, I. (2011b). Natural kinds and concepts: A pragmatist and methodologically naturalistic account. In J. Knowles \& H. Rydenfelt (Eds.), Pragmatism, science and naturalism (pp. 171-196). Berlin: Peter Lang Publishing.

Brigandt, I. (2011c). Philosophy of biology. In S. French \& J. Saatsi (Eds.), The Continuum Companion to the Philosophy of Science (pp. 246-267). London: Continuum Press.

Brigandt, I. (forthcoming). The dynamics of scientific concepts: The relevance of epistemic aims and values. In U. Feest \& F. Steinle (Eds.), Scientific concepts and investigative practice. Berlin: de Gruyter.

Brigandt, I., \& Griffiths, P. E. (2007). The importance of homology for biology and philosophy. Biology and Philosophy, 22, 633-641. 
Brigandt, I., \& Love, A. C. (2008). Reductionism in biology. In E. N. Zalta (Ed.), The Stanford Encyclopedia of Philosophy (Fall 2008 edition).

http://plato.stanford.edu/archives/fall2008/entries/reduction-biology

Brigandt, I., \& Love, A. C. (2010). Evolutionary novelty and the evo-devo synthesis: Field notes. Evolutionary Biology, 37, 93-99.

Cartwright, N. (1983). The truth doesn't explain much. In N. Cartwright (Ed.), How the laws of physics lie (pp. 44-53). Oxford: Oxford University Press.

Craver, C. F. (2005). Beyond reduction: Mechanisms, multifield integration and the unity of neuroscience. Studies in the History and Philosophy of Biological and Biomedical Sciences, 36, 373-395.

Craver, C. F. (2007). Explaining the brain: Mechanisms and the mosaic unity of neuroscience. Oxford: Oxford University Press.

Craver, C. F. (2009). Mechanisms and natural kinds. Philosophical Psychology, 22, 575-594.

Darden, L. (2005). Relations among fields: Mendelian, cytological and molecular mechanisms. Studies in History and Philosophy of Biological and Biomedical Sciences, 36, 349-371.

Darden, L. (2006). Reasoning in biological discoveries: Essays on mechanisms, interfield relations, and anomaly resolution. Cambridge: Cambridge University Press.

Darden, L., \& Maull, N. (1977). Interfield theories. Philosophy of Science, 44, 43-64.

Douglas, H. (2009). Science, policy, and the value-free ideal. Pittsburgh: University of Pittsburgh Press.

Dupré, J. (1993). The disorder of things: Metaphysical foundations of the disunity of science. Cambridge, MA: Harvard University Press. 
Fodor, J. (1974). Special sciences (or: The disunity of sciences as a working hypothesis). Synthese, 28, 97-115.

Gerhart, J. C., \& Kirschner, M. W. (2007). The theory of facilitated variation. Proceedings of the National Academy of Sciences USA, 104, 8582-8589.

Godfrey-Smith, P. (2009). Darwinian populations and natural selection. Oxford: Oxford University Press.

Grantham, T. A. (2004). Conceptualizing the (dis)unity of science. Philosophy of Science, 71, 133-155.

Griffiths, P. E., \& Stotz, K. (2007). Gene. In D. L. Hull \& M. Ruse (Eds.), The Cambridge Companion to the Philosophy of Biology (pp. 85-102). Cambridge: Cambridge University Press.

Hacking, I. (1983). Representing and intervening: Introductory topics in the philosophy of natural science. Cambridge: Cambridge University Press.

Hempel, C. G. (1965). Aspects of scientific explanation and other essays in the philosophy of science. New York: Free Press.

Hempel, C. G., \& Oppenheim, P. (1948). Studies in the logic of explanation. Philosophy of Science, 15, 135-175.

Hendrikse, J. L., Parssons, T. E., \& Hallgrímsson, B. (2007). Evolvability as the proper focus of evolutionary developmental biology. Evolution \& Development, 393-401.

Hull, D. L. (1974). Philosophy of biological science. Englewood Cliffs: Prentice-Hall.

Hüttemann, A., \& Love, A. C. (in press). Aspects of reductive explanation in biological science: Intrinsicality, fundamentality, and temporality. British Journal for the Philosophy of Science. 
Kellert, S. H., Longino, H. E., \& Waters, C. K. (2006). Introduction: The pluralist stance. In S. H. Kellert, H. E. Longino \& C. K. Waters (Eds.), Scientific pluralism (pp. vii-xxvix, Minnesota Studies in the Philosophy of Science, volume 19). Minneapolis: University of Minnesota Press.

Kirschner, M. W., \& Gerhart, J. C. (2005). The plausibility of life: Resolving Darwin's dilemma. New Haven: Yale University Press.

Kitcher, P. (1984). 1953 and all that: A tale of two sciences. The Philosophical Review, 93, 335373.

Kitcher, P. (1989). Explanatory unification and causal structure. In P. Kitcher \& W. C. Salmon (Eds.), Scientific explanation (pp. 410-505). Minneapolis: University of Minnesota Press.

Kitcher, P. (1999). Unification as a regulative ideal. Perspectives on Science, 7, 337-348.

Kourany, J. (2010). Philosophy of science after feminism. Oxford: Oxford University Press.

Laubichler, M., \& Wagner, G. P. (2001). How molecular is molecular developmental biology? A reply to Alex Rosenberg's Reductionism redux: Computing the embryo. Biology and Philosophy, 16, 53-68.

Lloyd, E. A. (1994). The structure and confirmation of evolutionary theory. Princeton: Princeton University Press.

Love, A. C. (2005). Explaining evolutionary innovation and novelty: A historical and philosophical study of biological concepts. Dissertation, University of Pittsburgh. http://etd.library.pitt.edu/ETD/available/etd-05232005-142007

Love, A. C. (2008). Explaining evolutionary innovations and novelties: Criteria of explanatory adequacy and epistemological prerequisites. Philosophy of Science, 75, 874-886. 
Love, A. C. (2009). Typology reconfigured: From the metaphysics of essentialism to the epistemology of representation. Acta Biotheoretica, 57, 51-57.

Love, A. C. (2010). Idealization in evolutionary developmental investigation: A tension between phenotypic plasticity and normal stages. Philosophical Transactions of the Royal Society of London B: Biological Sciences, 365, 679-690.

Love, A. C. (this issue). Interdisciplinary lessons for the teaching of biology from the practice of evo-devo. Science \& Education.

Machamer, P., Darden, L., \& Craver, C. F. (2000). Thinking about mechanisms. Philosophy of Science, 67, 1-25.

Maull, N. (1977). Unifying science without reduction. Studies in the History and Philosophy of Science, 8, 143-171.

Millstein, R. L. (2002). Are random drift and natural selection conceptually distinct? Biology and Philosophy, 17, 33-53.

Mitchell, S. D. (2002). Integrative pluralism. Philosophy of Science, 17, 55-70.

Mitchell, S. D. (2003). Biological complexity and integrative pluralism. Cambridge: Cambridge University Press.

Müller, G. B., \& Wagner, G. P. (2003). Innovation. In B. K. Hall \& W. M. Olson (Eds.), Keywords and concepts in evolutionary developmental biology (pp. 218-227). Cambridge, MA: Harvard University Press.

Nagel, E. (1949). The meaning of reduction in the natural sciences. In R. C. Stauffer (Ed.), Science and civilization (pp. 99-135). Madison: University of Wisconsin Press.

Nagel, E. (1961). The structure of science. New York: Harcourt, Brace, and World. 
Newman, S. A., \& Müller, G. B. (2005). Origination and innovation in the vertebrate limb skeleton: An epigenetic perspective. Journal of Experimental Zoology (Molecular and Developmental Evolution), 304B, 593-609.

Odenbaugh, J. (2003). Complex systems, trade-offs, and theoretical population biology: Richard Levin's "Strategy of model building in population biology" revisited. Philosophy of Science, 70, 1496-1507.

Okasha, S. (2006). Evolution and the levels of selection. Oxford: Oxford University Press.

Oppenheim, P., \& Putnam, H. (1958). Unity of science as a working hypothesis. In H. Feigl, M. Scriven \& G. Maxwell (Eds.), Concepts, theories, and the mind-body problem (pp. 3-36, Minnesota Studies in the Philosophy of Science, volume 2). Minneapolis: University of Minnesota Press.

Robert, J. (2004). Embryology, epigenesis, and evolution: Taking development seriously. Cambridge: Cambridge University Press.

Rosenberg, A. (1994). Instrumental biology or the disunity of science. University of Chicago Press: Chicago.

Ruse, M. (1971). Reduction, replacement, and molecular biology. Dialectica, 25, 39-72.

Salmon, W. C. (1971). Statistical explanation and statistical relevance. Pittsburgh: Pittsburgh University Press.

Salmon, W. C. (1984). Scientific explanation and the causal structure of the world. Princeton: Princeton University Press.

Sarkar, S. (1998). Genetics and reductionism. Cambridge: Cambridge University Press.

Schaffner, K. F. (1969). The Watson-Crick model and reductionism. British Journal for the Philosophy of Science, 20, 325-348. 
Schaffner, K. F. (1976). Reductionism in biology: Prospects and problems. In R. S. Cohen \& A. Michalos (Eds.), Proceedings of the 1974 biennial meeting of the Philosophy of Science Association (pp. 613-632). Dordrecht: Reidel.

Sober, E. (1980). Evolution, population thinking, and essentialism. Philosophy of Science, 47, 350-383.

Sober, E. (2008). Evidence and evolution: The logic behind the science. Cambridge: Cambridge University Press.

Stotz, K. (2006a). Molecular epigenesis: Distributed specificity as a break in the central dogma. History and Philosophy of the Life Sciences, 28, 527-544.

Stotz, K. (2006b). With 'genes' like that, who needs an environment? Postgenomics's argument for the 'ontogeny of information’. Philosophy of Science, 73, 905-917.

Vance, R. E. (1996). Heroic antireductionism and genetics: A tale of one science. Philosophy of Science, 63, S36-S45.

Wagner, G. P. (2000). What is the promise of developmental evolution? Part I: Why is developmental biology necessary to explain evolutionary innovations? Journal of Experimental Zoology (Molecular and Developmental Evolution), 288, 95-98.

Walsh, D. M., Lewens, T., \& Ariew, A. (2002). The trials of life: Natural selection and random drift. Philosophy of Science, 69, 429-446.

Weber, M. (2005). Philosophy of experimental biology. Cambridge: Cambridge University Press.

Weisberg, M. (2006). Forty years of 'The strategy': Levins on model building and idealization. Biology and Philosophy, 21, 623-645.

Weisberg, M. (2007). Three kinds of idealization. The Journal of Philosophy, 104, 639-659. 
Wimsatt, W. C. (1976). Reductive explanations: A functional account. In R. S. Cohen \& A. Michalos (Eds.), Proceedings of the 1974 biennial meeting of the Philosophy of Science Association (pp. 671-710). Dordrecht: Reidel.

Wimsatt, W. C. (1979). Reduction and reductionism. In P. D. Asquith \& H. E. Kyburg (Eds.), Current research in philosophy of science: Proceedings of the PSA critical research problems conference (pp. 352-377). East Lansing: Philosophy of Science Association.

Wimsatt, W. C. (2007). Re-engineering philosophy for limited beings: Piecewise approximations to reality. Cambridge, MA: Harvard University Press.

Winther, R. G. (2006). Parts and theories in compositional biology. Biology and Philosophy, 21, 471-499.

Winther, R. G. (2011). Part-whole science. Synthese, 178, 397-427.

Woodward, J. (2003). Making things happen: A theory of causal explanation. Oxford: Oxford University Press. 